\title{
Striving to recruit: the difficulties of conducting clinical research on elderly care home residents
}

\author{
Arnold G Zermansky \\ David P Alldred \\ Duncan R Petty \\ David K Raynor
}

J R Soc Med 2007; 100:258-261

Elderly care home residents are a distinct group of over 440000 people in over 18000 homes in England. ${ }^{1}$ Their mortality, ${ }^{2}$ health status and needs ${ }^{3,4}$ are different from those of people of the same age living in their own homes. Indeed, their physical and/or mental frailty and multiple pathology is usually the reason why they live in a residential setting. They are so different in almost every way from people of the same age in their own homes that research outcomes on the latter are simply not valid for them and it is important to conduct research specifically on care home residents.

Conducting research on care home residents is beset with constraints and complexities that can impair progress. Careful planning and an extraordinary amount of time and resources are needed to overcome them. We recently completed a large randomized controlled trial of clinical medication review by a pharmacist of elderly residents of care homes. ${ }^{5}$ In the process we encountered and struggled with many these problems. We hope that, by highlighting the issues and pitfalls, we can help future researchers plan projects involving care home residents.

\section{SAMPLING AND CONSENT}

A representative population requires sampling a large number of homes. Care homes vary in size, in our study from those with only six residents to one with over 200 . They are unevenly distributed geographically. Some are purely nursing, some residential and some mixed. They vary in management type from large national groups like BUPA, through local authority owned, to individually owned and run. There is little evidence as to how these differences affect either the care of residents or their therapeutic needs. It is therefore important in designing a study to include multiple homes and consider stratifying by type and size. Unfortunately this increases sample size and the overall cost and workload of the project. The larger the number of homes studied, the greater the organizational and logistical workload. A large number of participants in one

Baines Wing University of Leeds, PO Box 214, Leeds, West Yorkshire LS2 9UT, UK

Correspondence to: Dr A Zermansky

Email: a.g.zermansky@leeds.ac.uk home, on the other hand, creates economies of scale but undermines external validity. Cluster randomization would assume a degree of uniformity between homes of the same type/size that cannot be justified.

Any clinical study of care home residents needs three 'layers' of cooperation. This is because the collaboration of the home and each resident's doctor is required as well as the consent of the resident. It is unusual for all residents of a home to share the same doctor or even practice. The agreement of several doctors may be needed for one home. Whilst it can be argued that refusal to participate by a care manager or the doctor for a consented patient is an infringement of the patient's rights, in reality it would be impossible to conduct research without their active involvement.

It is impossible and impolitic to work with residents without the collaboration of the manager and care staff. Identifying residents and gaining access to them requires their involvement. In the case of residents with cognitive or communication difficulties, care staff will help explain the project to the resident, and be on hand at interview. In homes belonging to larger organizations, the researcher may need approval from a more senior manager. Seeking agreement of the home manager is seldom achievable just by letter. They need to understand the project and the implications for their residents (for whom they have a quasiparental responsibility). They must be satisfied with the researcher's credentials and understand what is required of themselves and their staff. They may need an incentive to participate, which might be a fee for their time, or perhaps a benefit for the residents or the home itself. In our project we reviewed the medication of intervention patients. Many home managers saw this as valuable and agreed to participate for this reason. To achieve this level of understanding requires a personal approach, and a face-toface meeting is useful.

It is essential to discover whether each resident can give informed consent. For those who cannot give informed consent, Department of Health guidance ${ }^{6}$ states that assent should be sought, usually from a near relative. The Local Ethics Committee must be satisfied that the exclusion of people with cognitive impairment would be to the disadvantage of people with cognitive impairment generally, and that participants would not be 'exposed to tangible 
risk'. We initially sought the home manager's opinion of whether the resident would understand the project, and sought assent for those who would not. In practice our research assistants sometimes found that residents identified as able to give consent were unable to understand the project, and we therefore needed to seek assent for these too.

The requirement for valid informed consent includes describing the project in writing (using appropriate language), but also a face-to-face discussion, with opportunity to ask questions. The researcher must be satisfied that the patient understands what is being asked. The patient must then be allowed 24 hours to reconsider before signing the consent.

We intended that home managers would seek consent, as we thought that this would be least intimidating for patients. In fact most managers found this too complex and time consuming, and we had to adapt our project (and seek additional funding and personnel) so that a research assistant could see each patient. Some patients who seemed to understand the project when it was explained, had forgotten all about it by the following day. We then felt bound to seek assent for them. We ultimately achieved a consent rate of $42 \%$ of those initially judged able to give informed consent, in part because patients assumed to be mentally competent turned out not to be. We have read papers reporting much higher consent rates in care home residents, to the extent that we are bound to wonder about the quality of informed consent achieved. Perhaps readers who have achieved high consent rates in their research might share the details of their methods.

Seeking assent was simpler. We conducted this by letter, giving the relative the opportunity to discuss the project. In fact only a handful of relatives contacted us. We achieved an assent rate of $41 \%$ of those in whom consent was not possible. Those who did contact us generally expressed understandable concern about possibly upsetting their relative.

We needed the agreement of patients' doctors to examine medical records and to make recommendations for those the clinical pharmacist had interviewed. Although this was a simple process conducted by letter, a small number of practices declined to participate even though we had patients' consent. Failing to include one practice could lead to the 'loss' of consented patients. We lost 60 patients from four practices in this way. We had taken the precaution of seeking prior approval of the relevant Local Medical Committees and Health Authorities. Two practices felt we should have sought their consent before approaching patients. This would not have been possible, because we needed the patients' consent to have access to their doctors' identity. Whilst there is an ethical question about the right of doctors to refuse when patients have consented, we are aware that practices receive many requests to participate in research, and that such involvement consumes tangible resources that would otherwise be available for patient care. Some financial reimbursement might be helpful in such situations.

Our overall level of consent/assent was only $42 \%$ $(1163 / 2779)$. Surprisingly, many studies of care home residents (even in august journals) do not state the numbers who decline to participate - for example Nijs et al., ${ }^{7}$ Fossey et al., ${ }^{8}$ Winblad et al. ${ }^{9}$ and Law et al. ${ }^{10}$. This raises issues about the external validity of outcomes. It is likely and perfectly reasonable that the sickest and frailest residents might decline to be involved. This may mean that the potential benefit (or harm) of the intervention cannot be measured in the group that has most to gain.

\section{DATA COLLECTION}

GP medical records were, at the time of our research, in an interim phase between paper-based and electronic. While virtually all practices recorded long-term medication on computer, other clinical information may have been recorded in either medium. Incoming letters and reports from hospitals are almost all on paper, though most practices now scan all incoming mail. Scanned mail may be readable on the screen but the data within it cannot be searched for unless it has been coded by the practice- - a routine now commonplace in response to information needs under the new NHS GP Contract. ${ }^{11}$ As regards important diagnoses, the extent to which case notes were summarized varied and it was not safe to assume that the computerized summary was complete. The special situation of care home residents, who are seldom seen at the surgery, means that the records of consultations tend to be written on paper at the time and transcribed on to the computer subsequently (if the doctor is diligent). In our study we needed to collect data on hospital admissions, number of GP consultations and a health problem list to contextualize the patient's medication. [It is a requirement of the new GP Contract that repeat medicines be linked with their indication, but many GP computer systems did not have this facility at the time of data collection.] This data was collected by a clinical pharmacist, as it required someone able to interpret clinical information. It took an average of 18 minutes per patient to collect this information (not including travelling time).

There is little uniformity of recording health-related information in care homes, either in terms of what is recorded or how records are kept. The only fairly consistent documents are Medicines Administration Record Sheets ('MAR Sheets') issued by the dispensing pharmacy and the (statutory) accident book, which records the details of falls. Nursing homes usually record clinical monitoring, (e.g. blood pressure, glucose, weight and bowels) and 
residential homes have social care notes describing residents' daily activities. The home may also record communications and consultations with GPs. Some GPs hold the patient's paper record or a summary at the home. When designing a study it is important to consider whether retrospective data will be accessible, reliable and consistent. The format and collection of prospective data is amenable to planning, but will still rely heavily on staff diligence.

\section{INTERVIEWING}

Consultations with care home residents present difficulties for two main reasons: those relating to access to the resident, and communication problems within consultations. Care home residents have a short day. They rise late and spend a long time in personal care, dressing and breakfasting. Their lunch is early and many doze afterwards. Their evening meal and preparation for bed is also early. The time windows for interview are therefore limited. The interviews themselves need to be conducted in private, and this requires the resident to walk (slowly!) to their room or be taken there by members of staff. An interview is quite often the social highlight of the resident's week, and they are generally happy to talk - often at great length, and not necessarily on issues relevant to the interview.

Many care home residents have communication difficulties due to cognitive impairment (three quarters of the intervention group in our trial had some degree of cognitive impairment based on MMSE), stroke-induced dysphasia, hearing or visual impairment. In our study, 50\% of residents were unable to hold a meaningful consultation with the pharmacist, usually as a result of dementia. Overcoming communication problems is challenging and may necessitate the presence of a member of care staff or a relative.

Our study included 661 patients registered with 60 general practices and living in 97 homes. Each home was visited at least five times (by the research nurse and the research pharmacist). Many were visited far more times than this to seek consent, collect data and interview patients who were unavailable at previous visits, and occasionally to collect missing data. Each general practice was visited at least twice. These homes were distributed unevenly within the greater Leeds area with a radius of about nine miles from our University office. Conducting the study in five randomly ordered zones one at a time reduced travelling time.

\section{MORTALITY}

Death rate was a secondary endpoint of our study, so that patients who died during the six-month study period were included. Nonetheless, there cannot be any 'end data' on

\section{Box 1 Advice for clinical researchers in care homes}

(1) Include homes of different types, ownership and size. Consider stratification, but be aware of the increased numbers required for this.

(2) Take into account high mortality in sample calculations.

(3) Work in geographically contiguous areas to minimize travelling time.

(4) Obtaining consent (or assent) is complex and very time consuming.

(5) Obtain approval of other stakeholders, including where appropriate GPs, home managers and relatives.

(6) You may have to offer incentives to homes and practices.

(7) Take account of the high level of cognitive and communication impairment of this population. You cannot overestimate the time needed to conduct interviews with care home residents; multiple visits are inevitable.

(8) Data collection from GP notes requires time, professional skill, and technical skill with GP computer systems.

(9) Data collected retrospectively from care home records may be difficult to access and interpret.

(10) Take into account the huge professional time requirements when calculating costs of the project.

medicines, did not have illnesses or mental state or activities of daily living. The mortality of the group was high $(90$ from 661 in six months, 13.6\%), and this considerably reduced the number available for analysis in respect of endpoints other than death. There were no important losses of patients from the study for other reasons. Very few patients moved homes, and those who did were easily traceable and followed up.

\section{costs}

The difficulties described above inevitably increase the cost of a clinical trial in this population, and it is vital for researchers and funders to recognize this. We estimate from our own experience that a trial conducted in care homes costs at least three times as much as a similar one on patients who live in their own homes. ${ }^{12}$

\section{CONCLUSIONS}

We are convinced of the need for and value of research in care homes and hope that others might learn from our painfully acquired experience. We have aggregated our experience-based advice into a number of practical points (summarized in Box 1).

Conducting clinical research with elderly care home residents is vital, but it is also challenging, time consuming 
and very expensive. Recruitment is particularly complex. Researchers need to plan and resource their projects to take account of the huge time requirement, the expected loss of potential participants between identification and consent, and the very high mortality of this group. Day to day planning needs to take cognisance of geography and the life and work patterns of homes and practices. It is important to offer resources to homes and GP practices that recognize the time that their staff may take to facilitate the project. Not only will these factors add significantly to the cost of the project, there is also a major time element (especially around recruitment) that must be included in the project timetable. Finally, funders need to take account of the need for research in this group, and recognize its cost implications. Whether a simplified approach to consent might be acceptable in research to measure the efficacy of non-experimental professional interventions is an issue for debate.

\section{Competing interests None declared.}

Acknowledgments The residents and their carers, the nursing homes and general practice staff for their cooperation. Members of our research team who are not co-authors of this paper: Prof. Nick Freemantle, Dr Jo Eastaugh, Dr Peter Bowie, Denise Buttress and Sue Thornton. The Healthcare Foundation who funded the study.

\section{REFERENCES}

1 Commission for Social Care Inspection. Annual Report and Accounts 2005-6. London: The Stationery Office, 2006

2 Nimmo AW, Peterkin G, Coid DR. Mortality in general practice: Problems concerning deaths in institutions. Public Health 2006;120:671

3 Sinclair AJ, Gadsby R, Penfold S, Croxson SCM, Bayer AJ. Prevalence of diabetes in care home residents. Diabetes Care 2001;24:1066-8

4 Petty DR, Scrivener G. Prescribing patterns for patients in homes. Prescriber 1998;9:103-6

5 Zermansky AG, Alldred DP, Petty DR, et al. Clinical medication review by a pharmacist of elderly people living in care homesrandomized controlled trial. Age Ageing 2006;35:586-91

6 Department of Health. Reference Guide to Consent for Examination and Treatment. London: DoH, 2001

7 Nijs KAND, de Graaf C, Kok FJ, van Staveren WA. Effect of family style mealtimes on quality of life, physical performance, and body weight of nursing home residents: cluster randomized controlled trial. BMJ 2006;332:1180-1184

8 Fossey J, Ballard C, Juszczak E, et al. Effect of enhanced psychosocial care on antipsychotic use in nursing home residents with severe dementia: cluster randomized trial. BMJ 2006;332:756-61

9 Winblad B, Kilander L, Eriksson S, et al. Donepezil in patients with severe Alzheimer's disease: double-blind, parallel-group, placebocontrolled study. Lancet 2006;367:1057

10 Law M, Withers H, Morris J, Anderson F. Vitamin D supplementation and the prevention of fractures and falls: results of a randomized trial in elderly people in residential accommodation. Age Ageing 2006;35:482-6

11 British Medial Association. Investing in General Practice. The New General Medical Services Contract. London: BMA, 2003

12 Zermansky AG, Petty DR, Raynor DK, Freemantle N, Vail A, Lowe CJ. Randomized controlled trial of clinical medication review by a pharmacist of elderly patients receiving repeat prescriptions in general practice. BMJ 2001;323:1340-3 